\title{
Reflection of Elastic Waves from Plane Surface of a Half-space with Impedance Boundary Conditions
}

\author{
Baljeet Singh \\ Department of Mathematics, Post Graduate Government College, Sector-11, Chandigarh - 160 011, India \\ Email: bsinghgc11@gmail.com
}

\begin{abstract}
In the present work, a problem on the reflection of elastic waves at a plane surface of an elastic half-space is considered. The elastic half-space is assumed homogeneous and isotropic. The plane surface of half-space is subjected to impedance boundary conditions, where normal and tangential tractions are proportional to normal and tangential displacement times frequency, respectively. The reflection coefficients of reflected $P$ and $S V$ are obtained in closed form for incidence of $P$ or $S V$ waves. These reflection coefficients depend on the angle of incidence, impedance parameters and other material parameters. The material parameters of Diabase (dark-colored igneous rock), Limestone (sedimentary rock) and Gneiss (high grade metamorphic rock) are chosen to compute the reflection coefficients for certain ranges of angle of incidence and impedance parameters. The effect of impedance boundary on the reflection phenomena is shown graphically for three different rock materials (Diabase, Limestone and Gneiss).
\end{abstract}

Keywords: Elastic half-space; Impedance boundary conditions; Elastic waves; Reflection coefficients.

\section{Introduction}

The phenomenon of wave propagation in solids is of interest in various branches of engineering and physical sciences. In particular, the propagation of mechanical disturbances in solids is applicable in the fields of mineral and oil exploration, geophysical exploration and seismology. The phenomenon of mechanical disturbances in solids has a long history. Love [1] in his treatise of mathematical theory of elasticity discussed the early investigations on propagation of mechanical disturbances in elastic solids by great mathematicians Augustin-Louis Cauchy and Simeon Denis Poisson. Remarkable investigations on propagation of waves in solids by Lord Rayleigh, Horace Lamb and Augustus Edward Hough Love between years 1880 to 1910 have made the phenomenon of wave propagation in solids popular till today. Various applications of this phenomenon are discussed in texts by Bullen [2], Ewing et al. [3], Cagniard [4], Miklowitz [5], Achenbach [6], Ben-Menahem and Singh [7] and many more. A significant number of problems on reflection phenomenon of elastic waves at free surface and interfaces of different elastic media may be found in literature. Some problems of interest in different elastic media are given in references list [8-22].

Impedance boundary conditions are a linear combination of unknown functions and their derivatives prescribed on the boundary. Impedance boundary conditions are commonly used in various fields of physics like acoustics and electromagnetism. However, these boundary conditions are not used much in seismology. There exist a number of different boundaries in the Earth's interior. Basically, the contact between two solids is a very complex phenomenon. Generally, in case of seismic wave interactions with discontinuities an ideally welded contact is assumed which includes the continuity of appropriate displacement and stress components. This kind of first approximation should be extended. Therefore, it is appropriate to assume contact planes as very thin layers which lead to impedance-like boundary conditions. Tiersten [23] derived impedance-like boundary conditions to observe the effect of a thin layer of different material over an elastic half-space. Malischewsky [24] investigated the Rayleigh waves with TierstenâĂŹs impedance boundary conditions and obtained a secular equation. Godoy et al. [25] proved the existence and uniqueness of Rayleigh waves with impedance boundary conditions. Vinh and Hue [26] discussed the propagation of Rayleigh waves in an orthotropic and monoclinic half-space with impedance boundary conditions. Singh [27] considered a problem on Rayleigh wave propagation in an isotropic generalized thermoelastic solid 
half-space with impedance boundary conditions. Recently, Vinh and Xuan [28] studied the propagation of Rayleigh waves with impedance boundary condition and derived an exact formula for the velocity by using the complex function method. Using this formula, they also established the existence and uniqueness of the wave. However, problems on reflection of elastic waves with impedance boundary conditions are not studied much in literature.

In this paper, the reflection phenomenon of elastic waves at a plane surface of elastic half-space is studied, where the surface of half-space is subjected to impedance boundary conditions. The reflection coefficients of reflected $P$ and $S V$ waves are obtained for incident $P$ or $S V$ waves. The dependence of the reflection coefficients on impedance parameters at each angle of incidence is shown graphically for material parameters of Diabase, Limestone and Gneiss.

\section{Governing Equations of Linear Elasticity}

Following Ewing et al. [3], the governing equations of linear, isotropic and homogeneous elastic medium in absence of body forces are

(a) Constitutive equations

$$
\begin{gathered}
e_{i j}=\frac{1}{2}\left(u_{i, j}+u_{j, i}\right), \\
\sigma_{i j}=2 \mu e_{i j}+\lambda e_{k k} \delta_{i j},
\end{gathered}
$$

(b) Equations of motion

$$
\mu u_{i, j j}+(\lambda+\mu) u_{j, i j}=\rho \ddot{u}_{i},
$$

where $e_{i j}$ are components of the strain tensor, $\sigma_{i j}$ are components of the stress tensor, $u_{i}$ are components of the displacement vector, $\delta_{i j}$ is Kronecker delta, $\rho$ is the density of the medium, $\lambda, \mu$ are Lame's constants. Subscripts preceded by a comma denote partial differentiation with respect to the corresponding Cartesian coordinate. The superposed dot denotes the partial differentiation with respect to the time $t$.

We consider a linear, isotropic and homogeneous elastic medium in the undeformed state. We take the origin at plane surface and negative $y$ - axis normally into the half-space which is thus represented by $y<0$. Following Godoy [25] and Vinh and Hue [26], we assume that the surface $y=0$ is subjected to impedance boundary conditions, where normal and tangential tractions depend linearly on normal and tangential displacements times frequency, respectively. We choose the $x$-axis in the direction of propagation of waves.

Using the following Helmholtz's representations

$$
u_{1}=\frac{\partial \phi}{\partial x}-\frac{\partial \psi}{\partial y}, \quad u_{2}=\frac{\partial \phi}{\partial y}+\frac{\partial \psi}{\partial x},
$$

in the equation (3), we obtain the following equations in $x-y$ plane,

$$
\begin{gathered}
(\lambda+2 \mu)\left[\frac{\partial^{2} \phi}{\partial x^{2}}+\frac{\partial^{2} \phi}{\partial y^{2}}\right]=\rho \frac{\partial^{2} \phi}{\partial t^{2}} \\
\mu\left(\frac{\partial^{2} \psi}{\partial x^{2}}+\frac{\partial^{2} \psi}{\partial y^{2}}\right)=\rho \frac{\partial^{2} \psi}{\partial t^{2}}
\end{gathered}
$$

Following Ewing et al. [3], there exist two plane waves in $\mathrm{x}-\mathrm{y}$ plane namely longitudinal wave $(P$ wave $)$ and shear wave ( $S V$ wave) with speeds $v_{1}$ and $v_{2}$ given by

$$
v_{1}=\sqrt{\frac{\lambda+2 \mu}{\rho}}, \quad v_{2}=\sqrt{\frac{\mu}{\rho}} .
$$




\section{Reflection at a Plane Surface}

An incident $P$ or $S V$ wave travels in half-space $y<0$ making an angle $\theta_{0}$ with normal to the half-space and impinges at plane surface $y=0$. The energy of an incident wave is partitioned into two reflected waves, namely, $P$ and $S V$ waves as shown in Figure 1. The potentials representing the incident and reflected waves are expressed as

$$
\begin{aligned}
& \phi=A_{0} e^{i k_{1}\left(\sin \theta_{0} x+\cos \theta_{0} y-v_{1} t\right)}+A_{1} e^{i k_{1}\left(\sin \theta_{1} x-\cos \theta_{1} y-v_{1} t\right)}, \\
& \psi=B_{0} e^{i k_{2}\left(\sin \theta_{0} x+\cos \theta_{0} y-v_{2} t\right)}+B_{1} e^{i k_{2}\left(\sin \theta_{2} x-\cos \theta_{2} y-v_{2} t\right)} .
\end{aligned}
$$

Here, for incident $P$ wave, $B_{0}=0$ and for incident $S V$ wave, $A_{0}=0$.

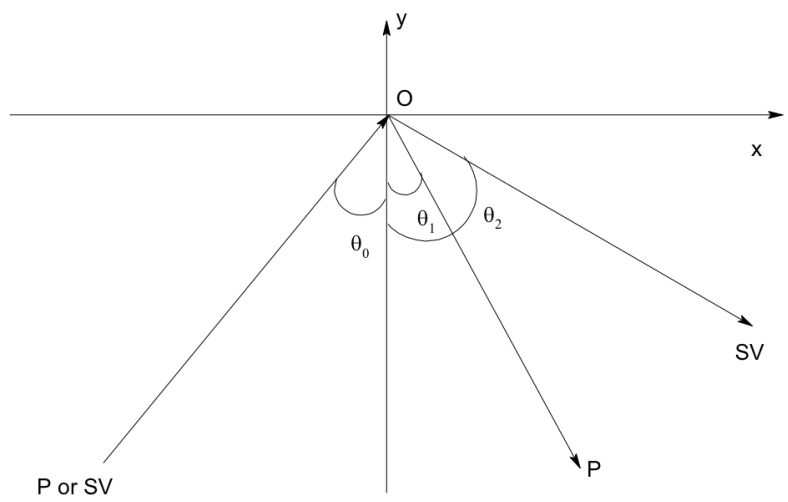

Figure 1. Geometry of the model showing incident and reflected waves.

The tangential force component $t_{y x}$ and normal force stress component $t_{y y}$ are proportional to tangential and normal displacement components times frequency, respectively, i.e.

$$
t_{y x}+\omega Z_{1} u_{1}=0, \quad t_{y y}+\omega Z_{2} u_{2}=0
$$

where

$$
\begin{gathered}
t_{y x}=\mu\left(2 \frac{\partial^{2} \phi}{\partial x \partial y}+\frac{\partial^{2} \psi}{\partial x^{2}}-\frac{\partial^{2} \psi}{\partial y^{2}}\right), \\
t_{y y}=\lambda\left(\frac{\partial^{2} \phi}{\partial x^{2}}-\frac{\partial^{2} \psi}{\partial x \partial y}\right)+(\lambda+2 \mu)\left(\frac{\partial^{2} \phi}{\partial y^{2}}+\frac{\partial^{2} \psi}{\partial x \partial y}\right),
\end{gathered}
$$

and $Z_{1}$ and $Z_{2}$ are the proportional coefficients called as impedance parameters. The traction free boundary conditions are recovered by setting $Z_{1}=0$ and $Z_{2}=0$.

At any boundary point and at any time, we also assume that the circular frequency of each reflected wave is equal to that of an incident wave, i.e.,

$$
k_{1} v_{1}=k_{2} v_{2}
$$

and the apparent wave number of every wave is equal, i.e.,

$$
k_{0} \sin \theta_{0}=k_{1} \sin \theta_{1}=k_{2} \sin \theta_{2} .
$$


where $k_{0}=k_{1}$ for incident $P$ wave and $k_{0}=k_{2}$ for incident $S V$ wave. Keeping in view of equations (13) and (14), the potentials given by equations (8) and (9) satisfy the boundary conditions (10) and we obtain the reflection coefficients of reflected $P$ and $S V$ waves in closed form as

$$
R_{1}=\frac{a_{12} b_{2}-a_{22} b_{1}}{a_{12} a_{21}-a_{11} a_{22}}, \quad R_{2}=\frac{a_{21} b_{1}-a_{11} b_{2}}{a_{12} a_{21}-a_{11} a_{22}},
$$

where for (a) Incident $P$ wave $\left(\theta_{0}=\theta_{1}\right)$ :

$$
\begin{aligned}
R_{1} & =\frac{A_{1}}{A_{0}}, \quad R_{2}=\frac{B_{1}}{A_{0}}, \\
a_{11} & =\sin \theta_{0}\left[2 \cos \theta_{0}+i\left(v_{1} / v_{2}\right) Z_{1}^{*}\right], \\
a_{12} & =\left[\left\{1-2\left(v_{2} / v_{1}\right)^{2} \sin ^{2} \theta_{0}\right\}+i Z_{1}{ }^{*} \sqrt{1-\left(v_{2} / v_{1}\right)^{2} \sin ^{2} \theta_{0}}\right]\left(v_{1} / v_{2}\right)^{2}, \\
b_{1} & =\sin \theta_{0}\left[2 \cos \theta_{0}-i\left(v_{1} / v_{2}\right) Z_{1}^{*}\right], \\
a_{21} & =-\left[\left(v_{1} / v_{2}\right)^{2}-2 \sin ^{2} \theta_{0}\right]-i\left(v_{1} / v_{2}\right) Z_{2}^{*} \cos \theta_{0}, \\
a_{22} & =\left(v_{1} / v_{2}\right) \sin \theta_{0}\left[2 \sqrt{1-\left(v_{2} / v_{1}\right)^{2} \sin ^{2} \theta_{0}}+i Z_{2}{ }^{*}\right], \\
b_{2} & =\left[\left(v_{1} / v_{2}\right)^{2}-2 \sin ^{2} \theta_{0}\right]-i\left(v_{1} / v_{2}\right) Z_{2}{ }^{*} \cos \theta_{0}, \\
Z_{1}{ }^{*} & =Z_{1} / \sqrt{\rho \mu}, \quad Z_{2}{ }^{*}=Z_{2} / \sqrt{\rho \mu},
\end{aligned}
$$

and for (b) Incident $S V$ wave $\left(\theta_{0}=\theta_{2}\right)$ :

$$
\begin{aligned}
R_{1} & =\frac{A_{1}}{B_{0}}, \quad R_{2}=\frac{B_{1}}{B_{0}}, \\
a_{11} & =\left(v_{2} / v_{1}\right) \sin \theta_{0}\left[2 \sqrt{1-\left(v_{1} / v_{2}\right)^{2} \sin ^{2} \theta_{0}}+i Z_{1}{ }^{*}\left(v_{1} / v_{2}\right)\right] \\
a_{12} & =\cos 2 \theta_{0}+i Z_{1}{ }^{*} \cos \theta_{0}, \\
b_{1} & =-\cos 2 \theta_{0}+i Z_{1}{ }^{*} \cos \theta_{0}, \\
a_{21} & =-\cos 2 \theta_{0}-i\left(v_{2} / v_{1}\right) Z_{2}{ }^{*} \sqrt{1-\left(v_{1} / v_{2}\right)^{2} \sin ^{2} \theta_{0}}, \\
a_{22} & =\sin 2 \theta_{0}+i Z_{2}{ }^{*} \sin \theta_{0}, \\
b_{2} & =\sin 2 \theta_{0}-i Z_{2}{ }^{*} \sin \theta_{0},
\end{aligned}
$$

These expressions for reflection coefficients reduce for the case of an elastic solid half-space with traction free boundary conditions when we set $Z_{1}=0$ and $Z_{2}=0$.

\section{Numerical Results and Discussion}

Following three different rock materials are chosen for numerical computations of reflection coefficients of reflected $P$ and reflected $S V$ for incidence of both $P$ and $S V$ waves:

$$
\begin{array}{llll}
\text { Diabase: } & \rho=2700 \mathrm{~kg} \cdot \mathrm{m}^{-3}, & E=90 \mathrm{GPa}, & \nu=0.2 \\
\text { Limestone: } & \rho=2400 \mathrm{~kg} \cdot \mathrm{m}^{-3}, & E=70 \mathrm{GPa}, & \nu=0.3 \\
\text { Gneiss: } & \rho=2800 \mathrm{~kg} \cdot \mathrm{m}^{-3}, & E=60 \mathrm{GPa}, & \nu=0.24
\end{array}
$$

The expressions for Lame's constants $\lambda$ and $\mu$ in terms of Young's Modulus $E$ and Poisson ratio $\nu$ are as

$$
\lambda=\frac{\nu E}{(1+\nu)(1-2 \nu)}, \quad \mu=\frac{E}{2(1+\nu)} .
$$




\subsection{Incident $\mathbf{P}$ Wave}

For incidence of $P$ wave from plane surfaces of diabase, limestone and gneiss, the variations of the reflection coefficients of reflected $P$ and $S V$ waves are shown graphically against the angle of incidence in figures 2 to 4 by solid $\left(Z_{1}=0, Z_{2}=0\right)$, small dashed $\left(Z_{1}=-100 \mathrm{MPa}, Z_{2}=-100 \mathrm{MPa}\right)$ and long dashed lines $\left(Z_{1}=50 \mathrm{MPa}, Z_{2}=50 \mathrm{MPa}\right)$. For $Z_{1}=0, Z_{2}=0$, the maximum values of reflection coefficients of reflected $P$ wave are one at normal incidence $\left(\theta_{0}=0^{\circ}\right)$ and at grazing incidence $\left(\theta_{0}=90^{\circ}\right)$ and the minimum values of reflection coefficients of reflected $P$ wave are found at angles of incidence $\theta_{0}=50^{\circ}, \theta_{0}=67^{\circ}$ and $\theta_{0}=57^{\circ}$ in diabase, limestone and gneiss, respectively. For $Z_{1}=0, Z_{2}=0$, the minimum values of reflection coefficients of reflected $S V$ are observed zero at normal and grazing incidences and the maximum values of reflection coefficients of reflected $S V$ wave are found at angles of incidence $\theta_{0}=43^{\circ}, \theta_{0}=47^{\circ}$ and $\theta_{0}=44^{\circ}$ in diabase, limestone and gneiss, respectively. The solid line variations $\left(Z_{1}=0, Z_{2}=0\right)$ in figures 2 to 4 correspond to those for traction free boundary and agree fairly with the existing established results in literature [ref. 3-7]. The comparison of different variations for different sets of impedance parameters in figures 2 to 4 shows the influence of impedance boundary on reflection coefficients of reflected $P$ and $S V$ waves in diabase, limestone and gneiss at each angle of incident except normal and grazing incidence. However, this effect is observed maximum at angles of incidence near to grazing incidence.

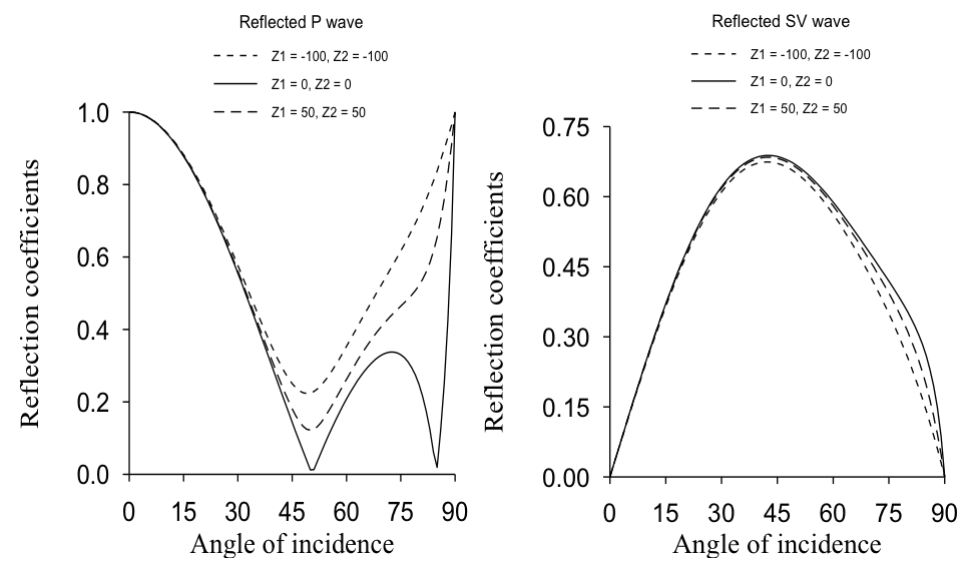

Figure 2. Variations of the reflection coefficients of reflected $\mathrm{P}$ and SV waves in diabase against the angle of incidence of $\mathrm{P}$ wave for different sets of impedance parameters.

In case of limestone, the variations of the reflection coefficients of reflected $P$ and $S V$ waves are shown in figure 5 against the impedance parameter $Z_{1}$ varying from $-100 M P a$ to $100 M P a$ when $Z_{2}=0$ and for angles of incidence $\theta_{0}=0^{\circ}, 30^{\circ}, 45^{\circ}, 60^{\circ}$ and $90^{\circ}$. For the range $-100 \leq Z_{1} \leq 100$, the reflection coefficient of $P$ wave is one at $\theta_{0}=0^{\circ}$ and $\theta_{0}=90^{\circ}$ as shown by solid line. For angles of incidences $\theta_{0}=30^{\circ}, 45^{\circ}$ and $60^{\circ}$, reflection coefficient of reflected $P$ wave is maximum at $Z_{1}=-100$ and $Z_{1}=100$ and attain minimum values $0.7043,0.4369$ and 0.2332 at $Z_{1}=0$ as shown by solid lines with center symbols as star, oplus and otimes, respectively. However, the reflection coefficient of reflected $S V$ wave is minimum at $Z_{1}=-100$ and $Z_{1}=100$ and attain maximum values at $Z_{1}=0$. Similar variations of the reflection coefficients of reflected $P$ and $S V$ waves are also shown against the impedance parameter $Z_{2}$ varying from -100 to 100 in Figure 6 , when $Z_{1}=0$ and for angles of incidence $\theta_{0}=0^{\circ}, 30^{\circ}, 45^{\circ}, 60^{\circ}$ and $90^{\circ}$. The comparison of different variations in Figures 5 and 6 shows the influence of impedance boundary on reflection coefficients of reflected $P$ and $S V$ waves at different angles of incidence. In diabase, limestone and gneiss, the variations of reflection coefficients of reflected $P$ and $S V$ waves are also shown against the impedance parameters $Z_{1}$ varying from -100 to 100 in Figure 7 , when $Z_{2}=0$ and $\theta_{0}=45^{\circ}$. The variations of the reflection coefficients of reflected $P$ and $S V$ waves in diabase, limestone and gneiss are also shown against the impedance parameter $Z_{2}$ varying from -100 to 100 in Figure 8 , when $Z_{1}=0$ and 

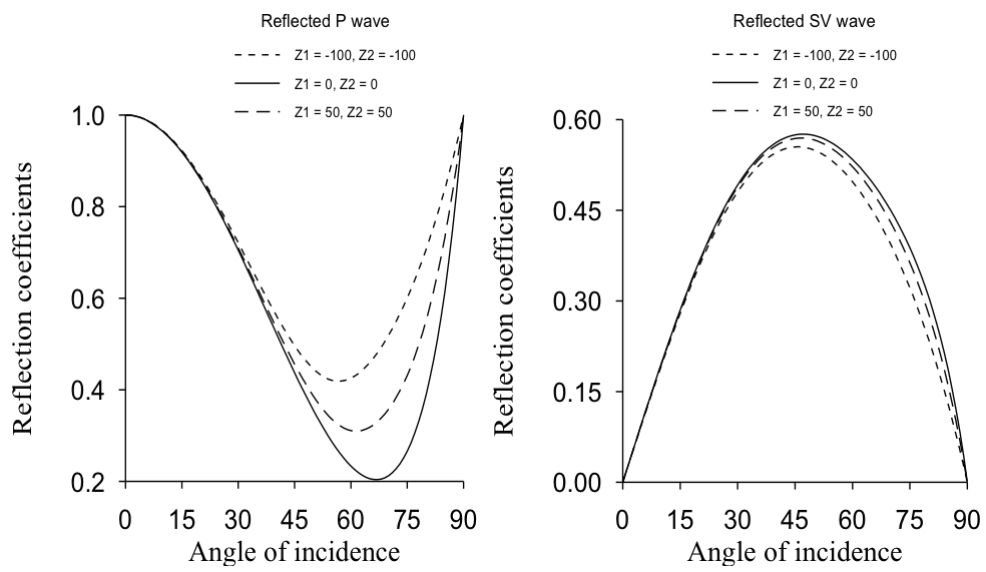

Figure 3. Variations of the reflection coefficients of reflected P and SV waves in limestone against the angle of incidence of $\mathrm{P}$ wave for different sets of impedance parameters.
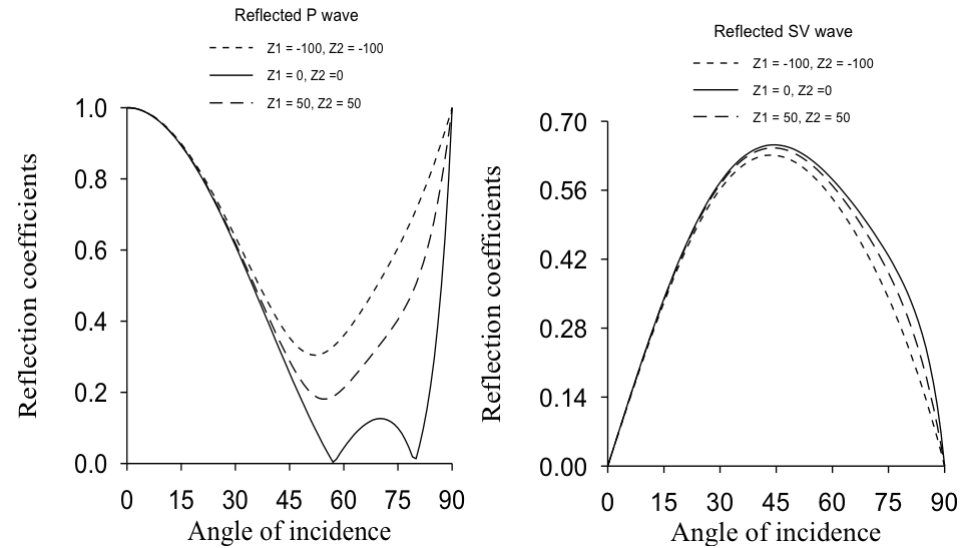

Figure 4. Variations of the reflection coefficients of reflected $\mathrm{P}$ and SV waves in gneiss against the angle of incidence of $\mathrm{P}$ wave for different sets of impedance parameters.
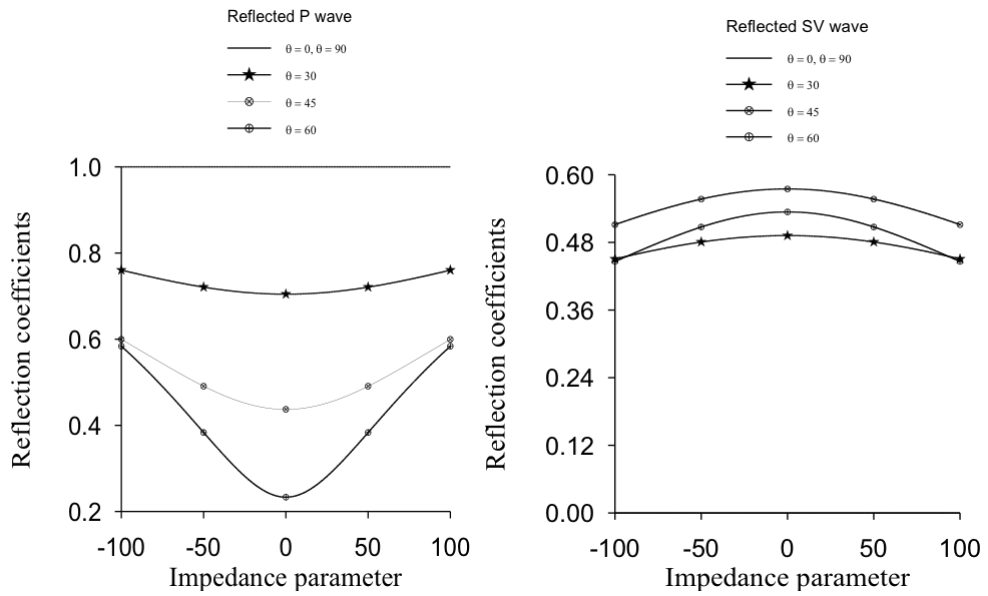

Figure 5. Variations of the reflection coefficients of reflected $\mathrm{P}$ and $\mathrm{SV}$ waves in limestone against the impedance parameter $Z_{1}$ for different angles on incidence of $\mathrm{P}$ wave when $Z_{2}=0$ 

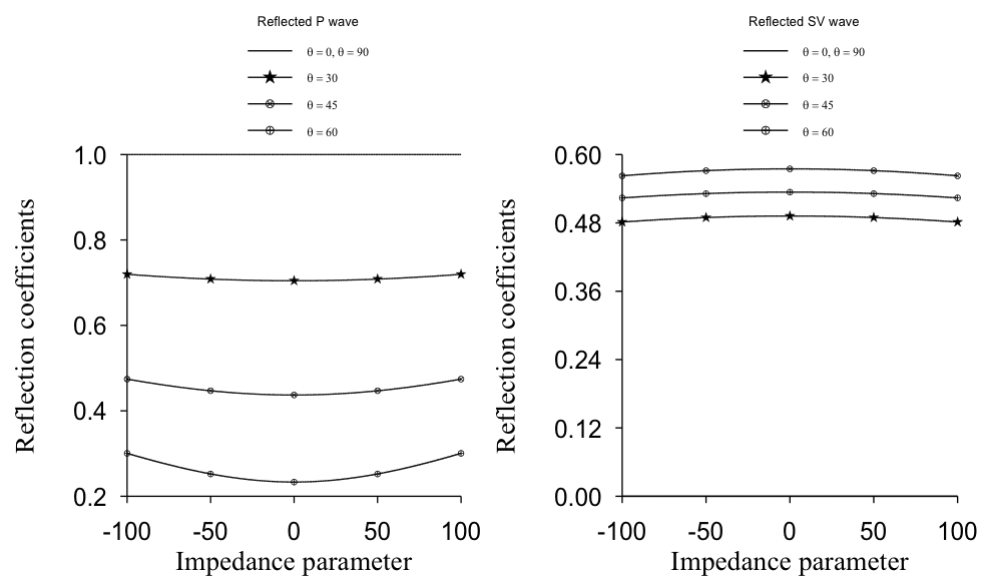

Figure 6. Variations of the reflection coefficients of reflected $\mathrm{P}$ and $\mathrm{SV}$ waves in limestone against the impedance parameter $Z_{2}$ for different angles on incidence of $\mathrm{P}$ wave when $Z_{1}=0$.

$\theta_{0}=45^{\circ}$. The comparison of different variations in Figures 7 and 8 shows the influence of impedance boundary at angle of incidence $\theta_{0}=45^{\circ}$ in three different rock materials.
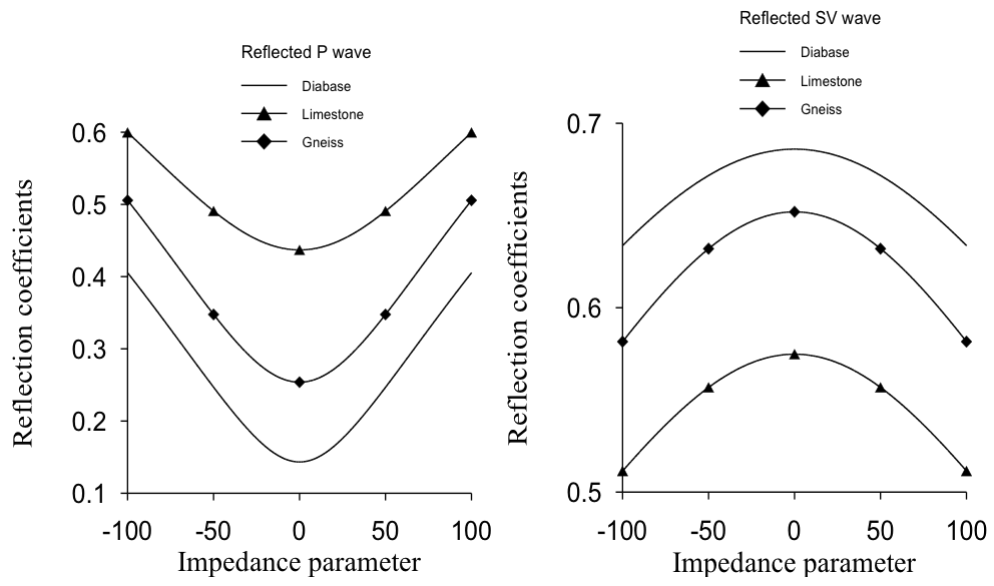

Figure 7. Variations of the reflection coefficients of reflected $\mathrm{P}$ and $\mathrm{SV}$ waves in diabase, limestone and gneiss against the impedance parameter $Z_{1}$ for angle $\theta=45^{\circ}$ of incident $\mathrm{P}$ wave when $Z_{2}=0$.

\subsection{Incident SV Wave}

For incident $S V$ wave, the variations of the reflection coefficients of reflected $P$ and $S V$ waves in diabase, limestone and gneiss, are shown graphically against the angle of incidence in Figures 9 to 11 for three different sets of impedance parameters $\left(Z_{1}=0, Z_{2}=0\right),\left(Z_{1}=-100, Z_{2}=-100\right)$ and $\left(Z_{1}=50, Z_{2}=50\right)$ by solid, small dashed and long dashed lines, respectively. For $Z_{1}=0, Z_{2}=0$ (traction free boundary), the minimum values of reflection coefficients of reflected $P$ are zero at normal incidence $\left(\theta_{0}=0^{\circ}\right)$ and the maximum values of reflection coefficients of reflected $P$ wave are found at angles of incidence $\theta_{0}=37^{\circ}$, $\theta_{0}=32^{\circ}$ and $\theta_{0}=35^{\circ}$ in diabase, limestone and gneiss, respectively. Beyond these critical angles, the total reflection takes place. $\left(Z_{1}=0, Z_{2}=0\right)$, the maximum values of reflection coefficients of reflected $S V$ are observed one at normal incidence and the minimum values of reflection coefficients of reflected $S V$ wave are observed at $\theta_{0}=28^{\circ}, \theta_{0}=29^{\circ}$ and $\theta_{0}=29^{\circ}$ in diabase, limestone and gneiss, respectively. 

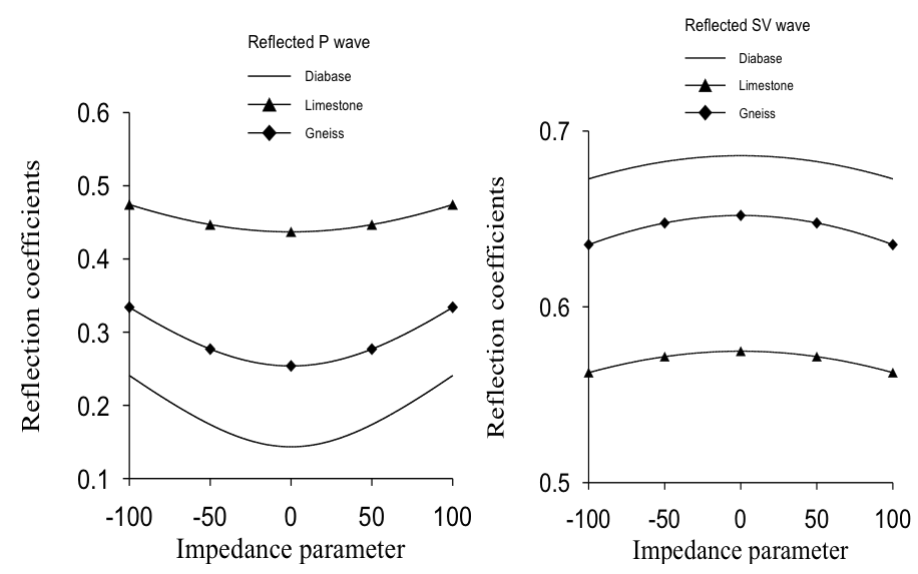

Figure 8. Variations of the reflection coefficients of reflected $\mathrm{P}$ and $\mathrm{SV}$ waves in diabase, limestone and gneiss against the impedance parameter $Z_{2}$ for angle $\theta=45^{\circ}$ of incident $\mathrm{P}$ wave when $Z_{1}=0$.

The comparison of different variations in figures 9 to 11 shows the significant influence of impedance boundary reflection coefficients of reflected $P$ and $S V$ waves in diabase, limestone and gneiss at each angle of incident except normal incidence. The variations of the reflection coefficients of reflected $P$ and
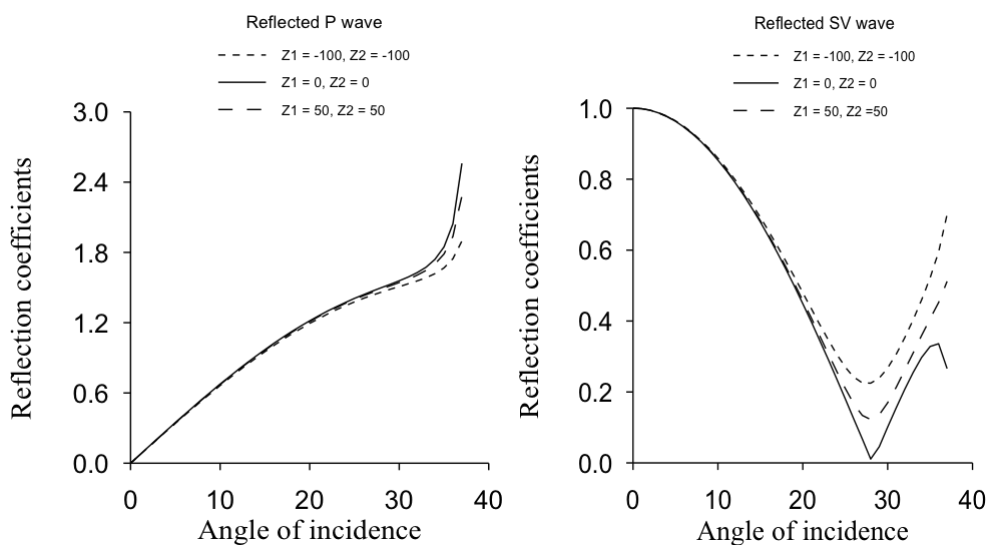

Figure 9. Variations of the reflection coefficients of reflected $\mathrm{P}$ and SV waves in diabase against the angle of incidence of SV wave for different sets of impedance parameters.

$S V$ waves in limestone are shown in Figure 12 against the impedance parameter $Z_{1}$ varying from -100 to 100 when $Z_{2}=0$ and for angles of incidence $\theta_{0}=0^{\circ}, 10^{\circ}, 20^{\circ}$ and $30^{\circ}$. For the range $-100 \leq Z_{1} \leq 100$, the reflection coefficient of $P$ wave is zero at $\theta_{0}=0^{\circ}$ as shown by solid line along horizontal axis in Figure 12. For angles of incidences $\theta_{0}=10^{\circ}, 20^{\circ}$ and $30^{\circ}$, the reflection coefficient of reflected $P$ wave is minimum at $Z_{1}=-100$ and $Z_{1}=100$ and attain maximum values $0.6816,1.283$ and 2.0936 at $Z_{1}=0$ as shown by solid lines with center symbols as star, oplus and otimes, respectively. However, the reflection coefficient of reflected $S V$ wave is maximum at $Z_{1}=-100$ and $Z_{1}=100$ and attain minimum values at $Z_{1}=0$. For the case of limestone, the variations of the reflection coefficients of reflected $P$ and $S V$ waves are also shown against the impedance parameter $Z_{2}$ varying from -100 to 100 in Figure 13 , when $Z_{1}=0$ and for angles of incidence $\theta_{0}=0^{\circ}, 10^{\circ}, 20^{\circ}$ and $30^{\circ}$. The comparison of different variations in figures 12 and 13 shows the effects of impedance boundary on reflection coefficients of reflected $P$ and $S V$ waves at different angles of incidence. For diabase, limestone and gneiss, the variations of reflection coefficients of reflected $P$ and $S V$ waves are also shown against the impedance parameters $Z_{1}$ varying from -100 to 100 in Figure 14, when $Z_{2}=0$ and $\theta_{0}=15^{\circ}$. The variations of the reflection coefficients of reflected 

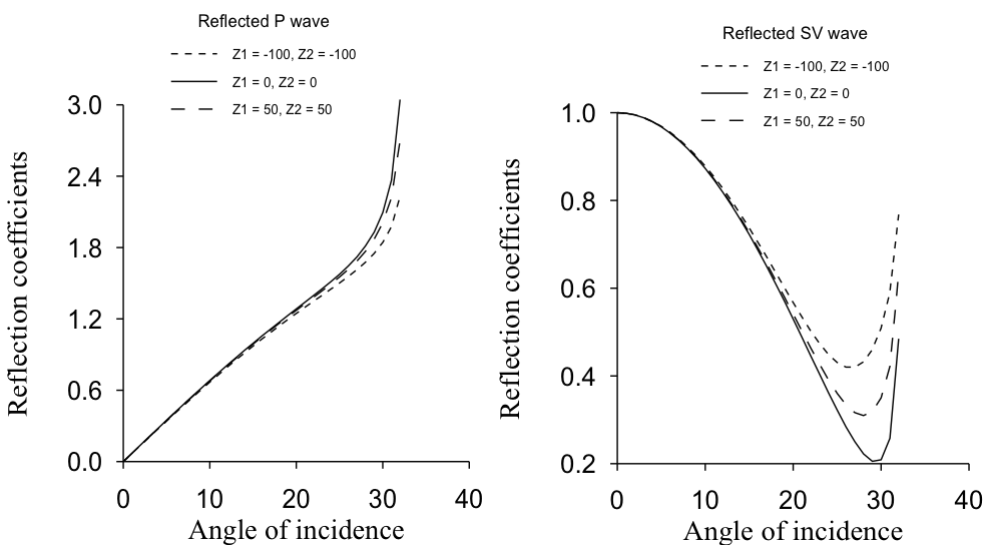

Figure 10. Variations of the reflection coefficients of reflected $\mathrm{P}$ and SV waves in limestone against the angle of incidence of SV wave for different sets of impedance parameters.
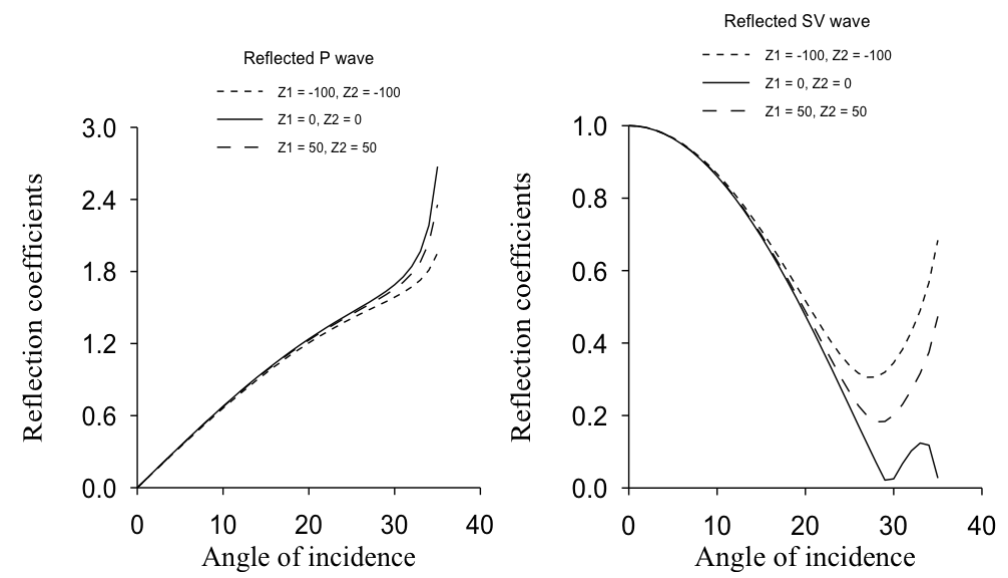

Figure 11. Variations of the reflection coefficients of reflected $\mathrm{P}$ and $\mathrm{SV}$ waves in gneiss against the angle of incidence of SV wave for different sets of impedance parameters.
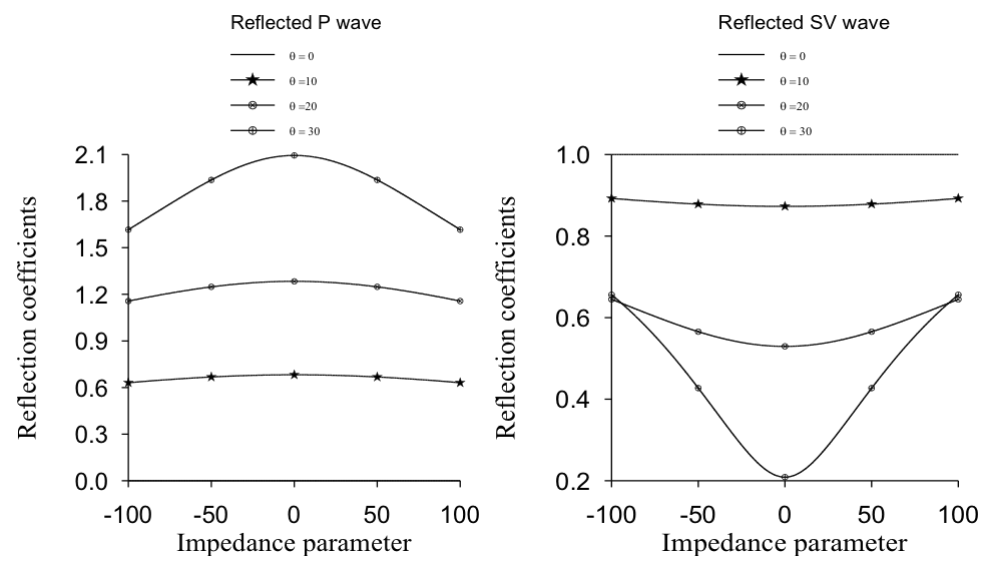

Figure 12. Variations of the reflection coefficients of reflected $\mathrm{P}$ and $\mathrm{SV}$ waves in limestone against the impedance parameter $Z_{1}$ for different angles on incidence of SV wave when $Z_{2}=0$.

$P$ and $S V$ waves in diabase, limestone and gneiss are also shown against the impedance parameter $Z_{2}$ varying from -100 to 100 in Figure 8 , when $Z_{1}=0$ and $\theta_{0}=15^{\circ}$. The comparison of different variations 

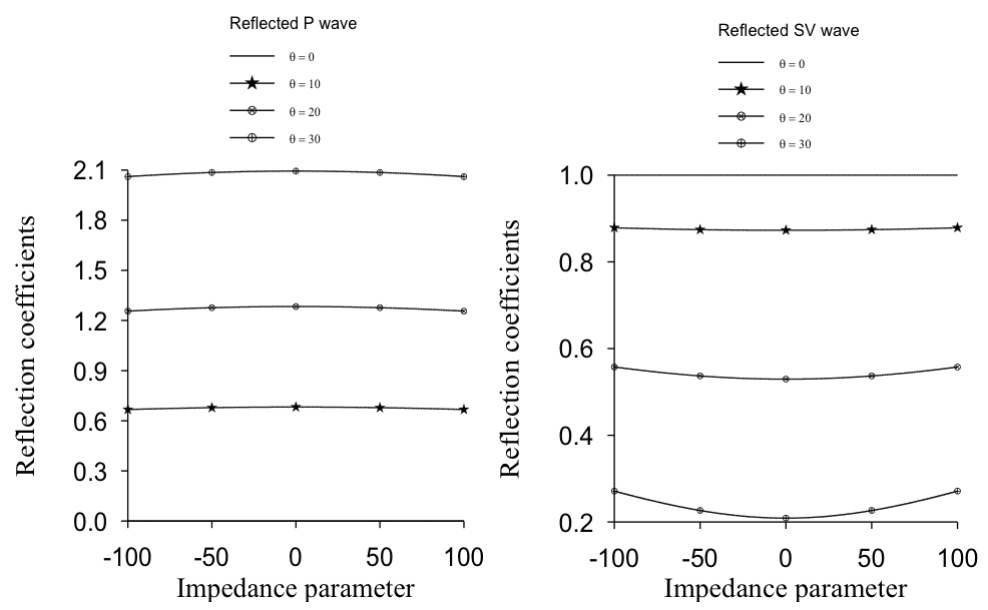

Figure 13. Variations of the reflection coefficients of reflected $\mathrm{P}$ and $\mathrm{SV}$ waves in limestone against the impedance parameter $Z_{2}$ for different angles on incidence of SV wave when $Z_{1}=0$.

in Figures 14 and 15 shows the impact of impedance boundary on reflection coefficients at $\theta_{0}=15^{\circ}$ in three different rock materials.
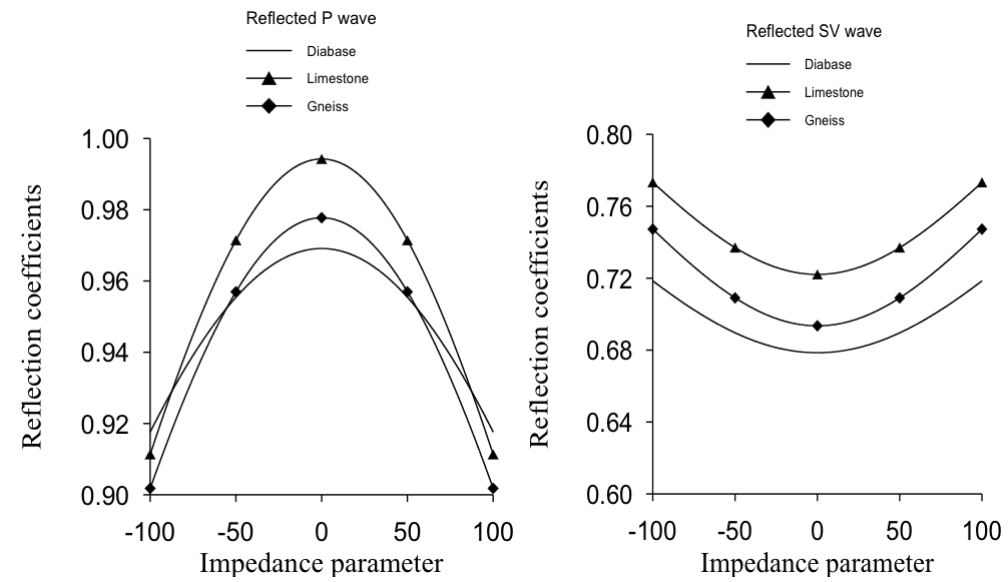

Figure 14. Variations of the reflection coefficients of reflected $\mathrm{P}$ and SV waves in diabase, limestone and gneiss against the impedance parameter $Z_{1}$ for angle $\theta=15 \circ$ of incident $\mathrm{SV}$ wave, when $Z_{2}=0$.

\section{Conclusions}

An elastic solid half-space is considered whose plane surface is subjected to impedance boundary conditions. A problem on reflection of $P$ and $S V$ waves is considered. The reflection coefficients of reflected $P$ and $S V$ waves are obtained in closed form. These coefficients are computed numerically for diabase, limestone and gneiss to show the impact of impedance boundary. From theory and numerical results, following observations are made:

(i) In diabase, limestone and gneiss, the reflection coefficient of reflected $P$ wave increases at each angle of incidence (except normal and grazing incidences) with the increase in $\left|Z_{1}\right|$ or $\left|Z_{2}\right|$. However, the reflection coefficient of reflected $S V$ wave decreases with the increase in $\left|Z_{1}\right|$ or $\left|Z_{2}\right|$.

(ii) The impact of impedance parameters is observed significant in range $40 \leq \theta_{0}<90$ for incidence of 

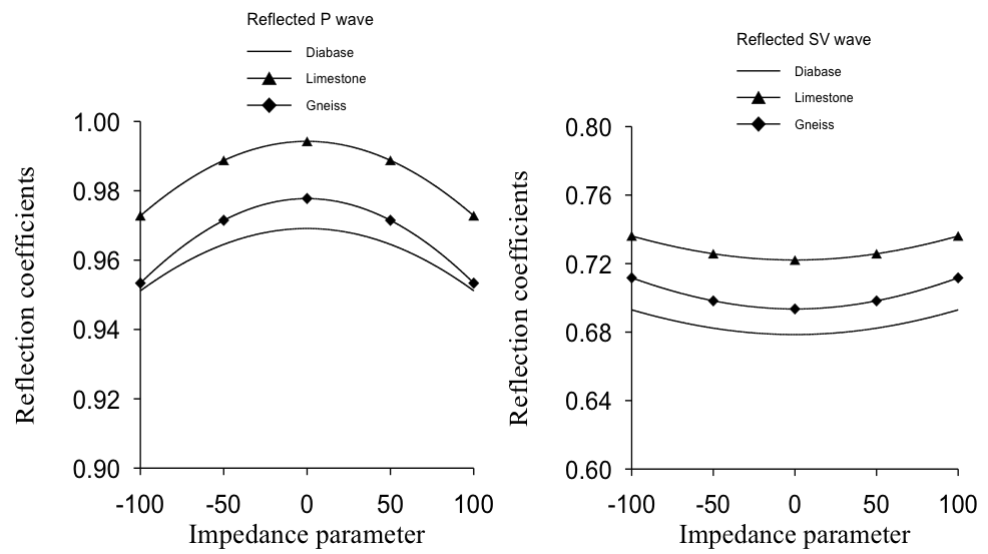

Figure 15. Variations of the reflection coefficients of reflected $\mathrm{P}$ and SV waves in diabase, limestone and gneiss against the impedance parameter $Z_{2}$ for angle $\theta=15 \circ$ of incident $\mathrm{SV}$ wave, when $Z_{1}=0$.

$P$ wave. However, for incidence of $S V$ wave, the maximum impact of impedance is observed at critical incidences.

(iii) For $Z_{1}=0, Z_{2}=0$, the numerical results correspond to those for traction free boundary and agree fairly with the established results found in literature.

(iv) The present numerical results may provide useful information to experimental scientists working in the field of wave propagation in solids.

\section{References}

1. A. E. H. Love, The mathematical theory of elasticity, 4th ed., New York, Dover Publications, Inc., 1944.

2. K. E. Bullen, An introduction to the theory of seismology, Cambridge University Press, 1963.

3. W. M. Ewing, W. S. Jardetzky and F. Press, Elastic waves in layered media, New York, McGraw-Hill Book Company, 1957.

4. L. Cagniard, Reflection and refraction of progressive waves, translated and revised by E. A. Flinn and C. H. Dix., New York McGraw-Hill Book Company, 1962.

5. J. Miklowitz, Elastic wave propagation, Applied Mechanics Surveys, Spartan Books, 1966.

6. J. D. Achenbach, Wave propagation in elastic solids, North Holland, 1973.

7. A. Ben-Menahem and S. J. Singh, Seismic waves and sources, Springer, 1981.

8. H. Deresiewicz, The effect of boundaries on wave propagation in a liquid-filled porous solid I. Reflection of plane waves at a free plane boundary (non-dissipative case, Bulletin of the Seismological Society of America, vol. 50, pp. 599-607, 1960.

9. D.G. Christie, Reflection of elastic waves from a free boundary, The London, Edinburgh, and Dublin Philosophical Magazine and Journal of Science, vol. 46, pp. 527-541, 1955.

10. V. R. Parfitt and A. C. Eringen, Reflection of plane waves from the flat boundary of a micropolar elastic halfâĂ Řspace, The Journal of the Acoustical Society of America, vol. 45, pp. 1258-1272, 1969.

11. A.N. Sinha and S.B. Sinha, Reflection of thermoelastic waves at a solid half-space with thermal relaxation, Journal of Physics of the Earth, vol. 22, pp. 237-244, 1974.

12. N. A. Haskell, Crustal reflection of plane P and SV waves, Journal of Geophysical Research, vol. 67, pp. 4751-4768, 1962.

13. M. Schoenberg and D. Censor, Elastic waves in rotating media, Quarterly of Applied Mathematics, vol. 31, pp. 115-125, 1973.

14. S. Dey and S. K Addy, Reflection of plane waves under initial stresses at a free surface, International Journal of Non-Linear Mechanics, vol. 12, pp. 371-381, 1977.

15. R. D. Borcherdt, ReflectionâĂTrefraction of general P-and type-I S-waves in elastic and anelastic solids, Geophysical Journal International, vol. 70, pp. 621-638, 1982.

16. R. S. Sidhu and S. J. Singh, Reflection of P and SV waves at the free surface of a prestressed elastic halfâĂ ̌rspace, The Journal of the Acoustical Society of America, vol. 76, pp. 594-598, 1984. 
17. S. I. Rokhlin, T. K. Bolland and L. Adler, Reflection and refraction of elastic waves on a plane interface between two generally anisotropic media, The Journal of the Acoustical Society of America, vol. 79, pp. 906-918, 1986.

18. R. W. Ogden and D. A. Sotiropoulos, Reflection of plane waves from the boundary of a pre-stressed compressible elastic half-space, IMA Journal of Applied Mathematics, vol. 61, pp. 61-90, 1998.

19. B. Singh, Reflection of P and SV waves from free surface of an elastic solid with generalized thermodiffusion, Journal of Earth System Science, vol. 114, pp. 159-168, 2005.

20. A. Chattopadhyay, P. Kumari and V. K Sharma, Reflection and refraction at the interface between distinct generally anisotropic half spaces for three-dimensional plane quasi-P waves, Journal of Vibration and Control, vol. 21, pp. 493-508, 2015.

21. M. Chatterjee, S. Dhua, A. Chattopadhyay, Quasi-P and quasi-S waves in a self-reinforced medium under initial stresses and under gravity, Journal of Vibration and Control, vol. 22, pp. 3965-3985, 2016.

22. P.J. Wei, Q. Tang and Y. Lia, Reflection and transmission of elastic waves at the interface between two gradient-elastic solids with surface energy, European Journal of Mechanics - A/Solids, vol. 52, pp. 54-71, 2015.

23. H. F. Tiersten, Elastic surface waves guided by thin films, Journal of Applied Physics, vol. 40, pp. 770-789, 1969.

24. P. G. Malischewsky, Surface waves and discontinuities, Elsevier, Amsterdam, 1987.

25. E. Godoy, M. Durn and J.-C. Ndlec, On the existence of surface waves in an elastic half-space with impedance boundary conditions, Wave Motion, vol. 49, pp. 585-594, 2012.

26. P.C. Vinh and T.T. Hue, Rayleigh waves with impedance boundary conditions in anisotropic solids, Wave Motion, vol. 51, pp. 1082-1092, 2014.

27. B. Singh, Rayleigh wave in a thermoelastic solid half-space with impedance boundary conditions, Meccanica, vol. 51, pp. 1135-1139, 2016.

28. P. C. Vinh and N. Q. Xuan, Rayleigh waves with impedance boundary condition: Formula for the velocity, existence and uniqueness, European Journal of Mechanics - A/Solids, vol. 61, pp. 180-185, 2017. 